
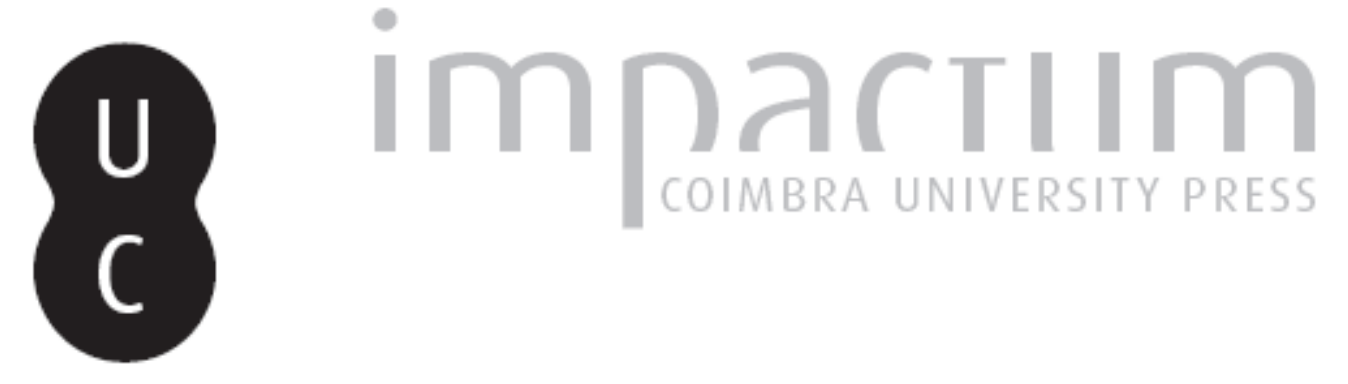

\title{
L'analogie platonicienne individu-État dans le De re publica de Cicéron: methode antilogique et fondement de la justice
}
Autor(es):
Renaud, François

Publicado por: Imprensa da Universidade de Coimbra

URL persistente:

URI:http://hdl.handle.net/10316.2/42187

DOI:

DOI:https://doi.org/10.14195/2183-4105_11_8

Accessed : $\quad$ 26-Apr-2023 11:44:00

A navegação consulta e descarregamento dos títulos inseridos nas Bibliotecas Digitais UC Digitalis, UC Pombalina e UC Impactum, pressupõem a aceitação plena e sem reservas dos Termos e Condições de Uso destas Bibliotecas Digitais, disponíveis em https://digitalis.uc.pt/pt-pt/termos.

Conforme exposto nos referidos Termos e Condições de Uso, o descarregamento de títulos de acesso restrito requer uma licença válida de autorização devendo o utilizador aceder ao(s) documento(s) a partir de um endereço de IP da instituição detentora da supramencionada licença.

Ao utilizador é apenas permitido o descarregamento para uso pessoal, pelo que o emprego do(s) título(s) descarregado(s) para outro fim, designadamente comercial, carece de autorização do respetivo autor ou editor da obra.

Na medida em que todas as obras da UC Digitalis se encontram protegidas pelo Código do Direito de Autor e Direitos Conexos e demais legislação aplicável, toda a cópia, parcial ou total, deste documento, nos casos em que é legalmente admitida, deverá conter ou fazer-se acompanhar por este aviso.

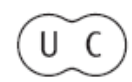




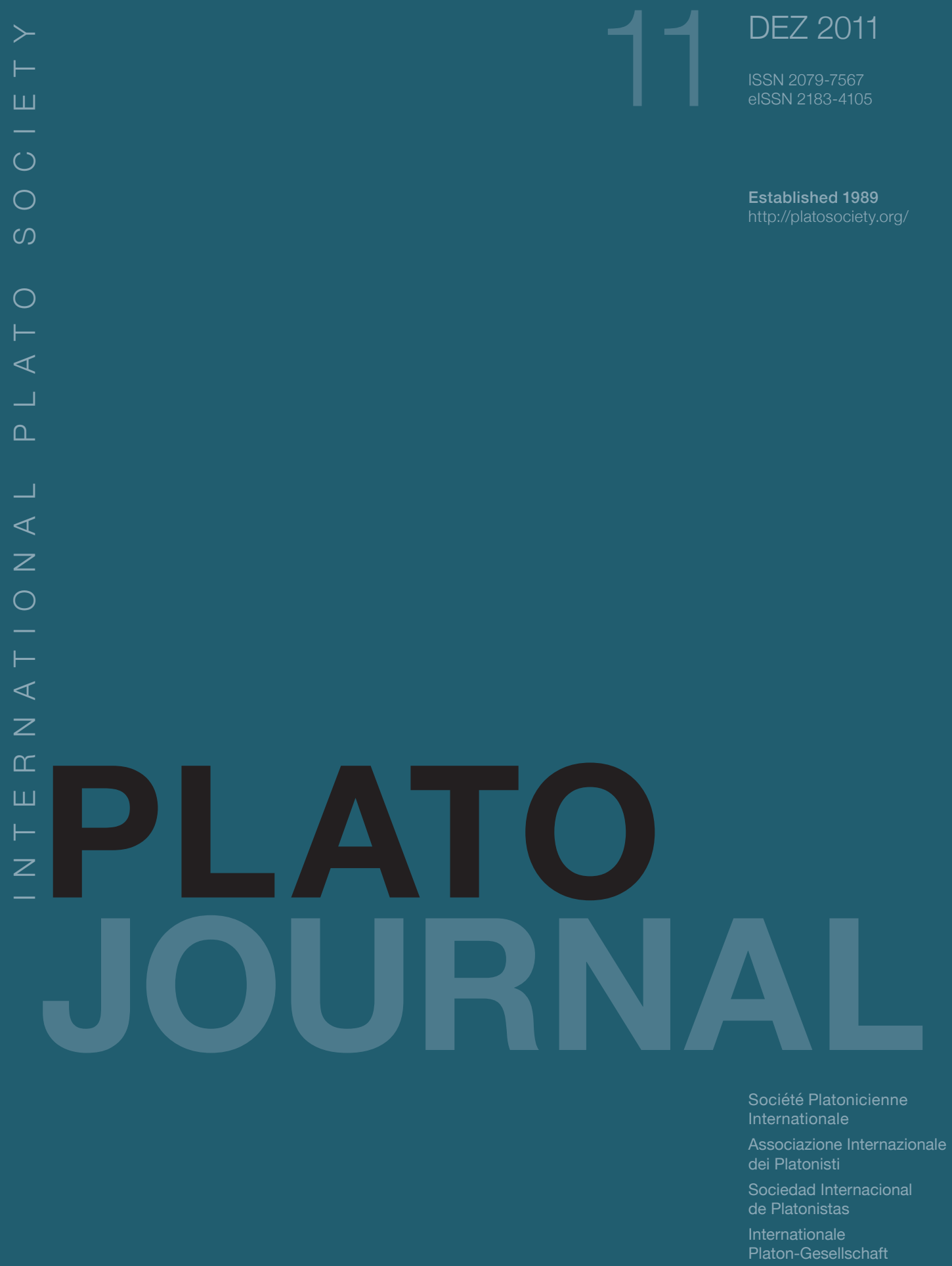


RENAUD, François, «L'analogie platonicienne individu-État dans le De re publica de Cicéron : méthode antilogique et fondement de la justice ».

\title{
L'ANALOGIE PLATONICIENNE INDIVIDU-ÉTAT DANS LE DE RE PUBLICA DE CICERON : METHODE ANTILOGIQUE ET FONDEMENT DE LA JUSTICE ${ }^{1}$
}

\author{
François RENAUD
}

Le De re publica de Cicéron est traditionnellement désigné comme le «pendant romain» de la République de Platon ${ }^{2}$. J'aimerais ici examiner à nouveaux frais la question du platonisme de Cicéron en me concentrant sur le livre III. Je tenterai de montrer, en partant du rapport entre méthode et contenu, que le platonisme de Cicéron réside notamment dans un aspect fondamental et pourtant largement ignoré par les commentateurs, soit dans un des aspects doctrinaux clés de la République: l'analogie individu-État, plus précisément la conception de la justice comme harmonie de l'âme et commandement de la raison. Cette brève étude sur la réception de Platon vise en dernière instance à contribuer à la question herméneutique et la question philosophique, et à celle du rapport entre elles.. Comment lire Platon ? Comment concevoir la justice ?

\section{1. État du texte, sources multiples, parallèles platoniciens}

${ }^{1}$ Je remercie l'auditoire au Symposium platonicum de Tokyo pour ses judicieuses remarques dont j'ai tenu compte dans la révision du texte, ainsi que le Conseil de recherches en sciences humaines du Canada pour son appui financier.

${ }^{2}$ Cette dénomination suit d'ailleurs certaines indications de l'auteur, voir p. ex. De re pub. II.i.10-11 : ut apud Platonem Socrates ipse finxero. Toutes les autres références au De re publica (éd. Powell 2006) sont indiquées sans le titre ; celles à la République de Platon (éd. Slings 2003) sont précédées de l'abréviation Rép. Les traductions françaises du De re publica sont celles de Bréguet (1980).

PLATO, The electronic Journal of the International Plato Society, n 11, 2011.

http://gramata.univ-paris1.fr/Plato/article100.html

(C) All rights of reproduction of any form reserved. 
RENAUD, François, «L'analogie platonicienne individu-État dans le De re publica de Cicéron : méthode antilogique et fondement de la justice ».

Quoique le texte du De re publica nous soit parvenu dans une condition fragmentaire ${ }^{3}$, il est néanmoins possible de discerner les grandes lignes de l'argumentation et de l'action dramatique. Composé entre 54 et 51 av. J.-C., le dialogue se divise en six livres, regroupés par paires, correspondant à trois journées de discussion. Vu l'absence de Cicéron comme personnage, la Correspondance et en particulier les Préambules (des livres I, III et V) permettent de mieux dégager l'intention de l'auteur. Tandis que le sujet général de l'ouvrage est « le meilleur régime et le meilleur citoyen » (de optimo statu civitatis et de optimo cive) ${ }^{4}$, le livre III $^{5}$ discute de la justice comme fondement de l'État. Quant à la forme, le livre est dominé par deux discours opposés prononcés par Philus et de Lélius, contre et pour la justice respectivement, suivis de celui de Scipion.

Les sources du De re publica sont multiples (platoniciennes, stoïciennes, péripatéticiennes), mais la recherche récente souligne avec raison la relative indépendance de Cicéron par rapport à celles-ci'. La présence de Platon dans le De re publica est complexe, allant de l'imitation à la rivalité et jusqu'à la critique explicite (notamment de la communauté des femmes et des enfants) ${ }^{7}$. Cette relative liberté se manifeste d'abord dans les procédés du dialogue comme genre littéraire. Comme dans le cas du De oratore, écrit à la même époque, les principaux interlocuteurs du De re publica (Scipion, Lélius, Philus) sont tous Romains, appartenant au passé ${ }^{8}$ et ayant l'insigne mérite d'allier l'expérience ancestrale romaine (mos maiorum) à la sagesse grecque (doctrina), notamment socratique9. Si Cicéron lui-même peut prétendre écrire avec autorité sur la politique, c'est aussi en vertu de son expérience tant pratique que théorique. Cette alliance entre théorie et pratique forme un thème sous-jacent de l'ouvrage sur lequel je reviendrai à la fin de mon analyse.

${ }^{3}$ Il nous a été transmis sous la forme d'un palimpseste incomplet (découvert en 1820 et publié en 1822), complété par quelques précieuses indications de la tradition indirecte (surtout Augustin et Lactance).

${ }^{4} Q u . f r$. III.5.1.

${ }^{5}$ Les fragments de ce livre totalisent 26 pages dans l'édition de Powell.

${ }^{6}$ Cf. De div. II.1.

${ }^{7}$ IV.v ; $c f$. p. ex. II.xi ; II.xxix.

${ }^{8}$ La date fictive du dialogue est 129 av. J.-C., peu avant la mort de Scipion.

${ }^{9}$ I.xxi ; $c f$. III.iii.

PLATO, The electronic Journal of the International Plato Society, n 11, 2011.

http://gramata.univ-paris1.fr/Plato/article100.html

(C) All rights of reproduction of any form reserved. 
RENAUD, François, «L'analogie platonicienne individu-État dans le De re publica de Cicéron : méthode antilogique et fondement de la justice ».

Outre le titre (traduction de $\pi 0 \lambda เ \tau \varepsilon i \alpha$ ) et la traduction libre de certains passages de la République ${ }^{10}$, les parallèles entre le De re publica et la République sont nombreux et variés. S'agissant plus particulièrement du livre III, Cicéron reprend notamment, en les adaptant, les aspects dialectiques et doctrinaux suivants (tirés surtout de Rép. I, II et IV) : (i) l'idée même d'un débat sur la nature et la valeur intrinsèque de la justice ${ }^{11}$; (ii) la méthode antilogique (in utramque partem) comme la plus adéquate pour la découverte de la véritée ${ }^{12}$; (iii) le rôle d'avocat du diable que Philus s'assigne à lui-même, à l'instar de Glaucon et d'Adimante, en prenant le parti contre la justice ${ }^{13}$; (iv) la nécessité de formuler une définition de la justice comme fondement de la cité idéale ; (v) les portraits contrastés de l'homme juste et de l'homme injuste ${ }^{14}$; (vi) enfin et surtout l'analogie platonicienne entre l'individu et l'État ${ }^{15}$, thèse dont la présence et les corollaires sont souvent ignorés ou sous-estimés par la recherche.

\section{Méthode antilogique, intention de l'auteur}

L'importance décisive du livre III dans l'économie du De re publica tient à son caractère éminemment philosophique. Il s'agit du seul livre du dialogue portant sur la justice comme fondement de l'État ${ }^{16}$. Comme le déclare Scipion à la fin du livre II, la discussion du lendemain (le livre III donc) doit décider de l'ensemble de l'entretien, en tranchant la question de savoir si un gouvernement peut subsister sans injustice ou si au contraire aucun gouvernement digne de ce

${ }^{10}$ Notamment la transition de la démocratie à la tyrannie : I.xliii-xliv ; Rép. 562c-563d.

${ }^{11}$ Sur l'importance de la recherche de la justice comme bien précieux, voir l'emprunt de l'expression : cum iustitiam quaeramus, rem multo omni auro cariorem (III.iv.10; cf. Rép. 336e7:

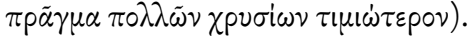

${ }^{12}$ neque sit ignota consuetudo tua contrarias in partis disserendi, quod ita facillume verum

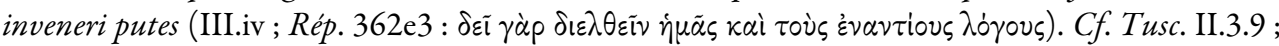
De fin. V.4.10.

${ }^{13}$ III.iv. Il ajoute même: sed iuventuti nostrae minime audiendus (III.xxi); Rép. 357a$367 a$.

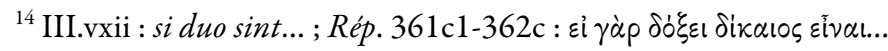

${ }^{15}$ Rép. 368e-369a.

${ }^{16}$ II.xxxix.

PLATO, The electronic Journal of the International Plato Society, n 11, 2011.

http://gramata.univ-paris1.fr/Plato/article100.html

(C) All rights of reproduction of any form reserved. 
RENAUD, François, « L'analogie platonicienne individu-État dans le De re publica de Cicéron : méthode antilogique et fondement de la justice ».

nom n'est possible sans la justice ${ }^{17}$. Cette question de fond se formule comme suit. La république repose-t-elle sur la nature ou la convention ? La justice est-elle bénéfique ou nuisible à qui la pratique ?

Philus prétend résumer le discours de Carnéade contre la justice que celui-ci tint lors de sa célèbre ambassade à Rome en 155. Dans ce discours, Carnéade aurait cherché à ruiner la justice en défendant les deux thèses principales suivantes : (i) la relativité des principes de justice et de droit (iustitia, ius), et (ii) l'opposition entre justice et sagesse ou prudence (sapientia). Ces thèses rappellent évidemment les théories sophistiques que Platon cherchait à renverser. Dans la rédaction du discours de Philus, Cicéron a recours à des arguments qui sont sans doute de Carnéade lui-même, quoiqu'il soit incertain s'il s'agit du contenu de la conférence tenue à Rome comme le prétend l'auteur romain ${ }^{18}$. Une importante différence, toutefois, s'impose d'emblée et oriente tout le débat: l'ordre des discours est inversé. Tandis que Carnéade finissait sa double conférence par le discours contre la justice (selon l'usage néo-académicien, le second discours est celui que l'orateur souhaite personnellement défendre ${ }^{19}$ ), Cicéron accorde le dernier mot à Lélius, défenseur de la justice et personnage jouissant par ailleurs d'un prestige politique supérieur à celui de Philus. L'intention cicéronienne est confirmée par la correction explicite, évoquée il y a un instant, que Scipion apporte à sa propre définition de res publica formulée au livre I. Par ailleurs, la défense de la justice, implicitement entreprise par Cicéron auteur, n'est nullement incompatible avec l'adhésion de celui-ci à la Nouvelle Académie $^{20}$; la définition retenue est celle qui devait lui apparaître la plus probable.

${ }^{17}$ II.xliv. C'est pourquoi Scipion modifiera, à la fin du livre III, la définition de res publica qu'il avait établie au livre I (en tant que coetus multitudinis iuris consensu et utilitatis communione sociatus, I.xxv) : sed, ut nunc ratio cogit, dicendum est plane nullam esse rem publicam (III.xxxi). Le fondement de la justice n'est pas la justice variable de la cité, mais la loi véritable, la justice de la raison, qui est universelle et immuable.

${ }^{18}$ Cf. Ferrary 1988, 361.

${ }^{19}$ Cf. Att. II.3.3.

${ }^{20}$ Cf. p. ex. Acad. 20.5 (éd. Plasberg); Glucker (1988) a soulevé la question de savoir si Cicéron est resté fidèle toute sa vie, y compris dans les années 50, à la Nouvelle Académie (de Philon) ou s'il se rattache plutôt, dans les années de composition du De republica, à Antiochus.

PLATO, The electronic Journal of the International Plato Society, n 11, 2011.

http://gramata.univ-paris1.fr/Plato/article100.html

(C) All rights of reproduction of any form reserved. 
RENAUD, François, « L'analogie platonicienne individu-État dans le De re publica de Cicéron : méthode antilogique et fondement de la justice ».

Résumons, très brièvement, le contenu des discours de Philus et de Lélius. Philus, avocat du diable donc, avance dans son discours contre la justice (contra iustitiam) que le droit (ius) est une convention qui n'a rien de naturel. La grande diversité des croyances et des coutumes ${ }^{21}$ prouve la relativité des normes morales. De plus, la justice et la sagesse (sapientia) s'opposent. Loin de commander de se soucier du bien d'autrui, la nature ordonne à tout être vivant de veiller à sa propre survie et aux moyens de l'assurer. Du point de vue individuel, seul l'insensé (stultus) peut préférer la vie de l'homme parfaitement juste à celle de l'homme parfaitement injuste, à plus forte raison si leurs réputations sont inversées ${ }^{22}$. Car il est toujours préférable de commettre impunément l'injustice ${ }^{23}$. Du point de vue des États, l'intérêt bien compris (sapientia) leur commande d'augmenter leurs richesses et d'agrandir leur territoire ${ }^{24}$. Lélius, pour sa part, renverse l'argumentation de Philus en faisant appel à la loi naturelle, loi véritable (vera lex), loi de la raison (recta ratio naturae), immuable et éternelle (constans, sempiterna), qu'elle soit respectée ou violée $e^{25}$. Cette loi de la raison est celle de Dieu (imperator omnium deus $)^{26}$. Toute domination n'est pas nécessairement injuste, si elle est fondée sur la vraie liberté, soit la maîtrise de soi et des passions ${ }^{27}$, et si elle est dans l'intérêt des petits ${ }^{28}$. L'histoire romaine offre des preuves et illustrations de l'existence de normes morales naturelles ${ }^{29}$.

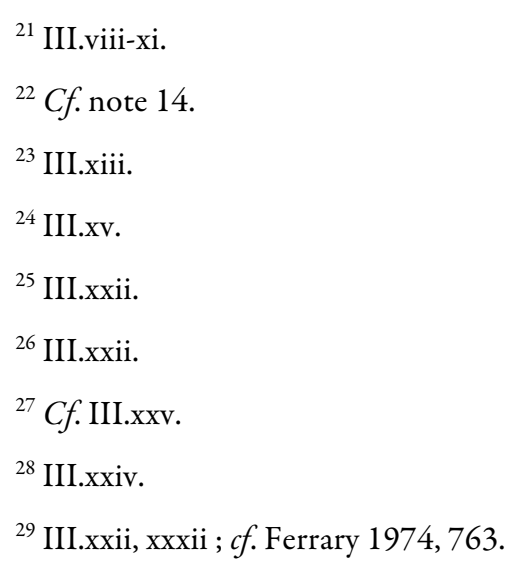

PLATO, The electronic Journal of the International Plato Society, n 11, 2011.

http://gramata.univ-paris1.fr/Plato/article100.html

(C) All rights of reproduction of any form reserved. 
RENAUD, François, «L'analogie platonicienne individu-État dans le De re publica de Cicéron : méthode antilogique et fondement de la justice ».

\section{Analogie individu-État et parties de l'âme}

Soucieux avant tout de souligner les différences entre le De republica et la République, la majorité des commentateurs voit la différence décisive dans l'accent cicéronien mis sur la justice dans les États (notamment l'empire romain et son histoire), alors que Platon s'intéresserait d'abord à la justice dans l'individu conçue comme harmonie intérieure. L'accent expressément politique, plutôt qu'éthique, de l'ouvrage de Cicéron en ferait même un «pendant inversé » de la République ${ }^{30}$. Cette interprétation dominante a tendance à sous-estimer et même à ignorer entièrement la présence, dans le De re publica, de l'analogie platonicienne entre l'âme et l'État et ses implications, dont la doctrine des parties de l'âme.

Premièrement, on a insuffisamment reconnu le fait que Philus autant que Lélius accepte expressément l'analogie entre l'individu et l'État. L'un et l'autre affirment que ce qui est vrai des individus l'est également des cités ou des peuples (quod in singulis, idem est in populis), que le principe de droit est identique dans les domaines public et privé ${ }^{31}$. Philus déclare en outre que l'opposition entre justice et sagesse apparaîtra plus nettement (inlustriora) dans les États que dans les individus ${ }^{32}$, ce qui rappelle le principe heuristique de Socrate dans la République consistant à rechercher d'abord la justice dans l'État, parce qu'il est plus facile de l'y voir ( $\dot{\alpha} \alpha \omega \nu \kappa \alpha \tau \alpha \mu \alpha \theta \varepsilon \tilde{\varepsilon})$ ), afin de la trouver ensuite dans l'individu ${ }^{33}$.

Deuxièmement, la doctrine des parties (partes) de l'âme, comme composante de l'analogie individu-État, est passée largement inaperçue. Cela est d'autant plus étonnant que Scipion et Lélius font allusion à la doctrine platonicienne des parties de l'âme de manière assez explicite. Certes, certaines références sont vagues ou ambiguës ${ }^{34}$, d'autres toutefois sont assez précises. Dans

${ }^{30}$ Cf. p. ex. Sharples 1986, 30 ; Hahn 1999, 183.

${ }^{31}$ III.xviii.9-10; cf. p. ex. Zetzel 1995, 29.

${ }^{32}$ III.xv.

${ }^{33}$ Rép. 368e7-369a1.

${ }^{34}$ Scipion p. ex. parle de la raison comme l'une des parties de l'âme sans toutefois faire références aux autres : quaeque pars animi mens vocatur (II.xl).

PLATO, The electronic Journal of the International Plato Society, n 11, 2011.

http://gramata.univ-paris1.fr/Plato/article100.html

(C) All rights of reproduction of any form reserved. 
RENAUD, François, « L'analogie platonicienne individu-État dans le De re publica de Cicéron : méthode antilogique et fondement de la justice ».

le discours de Lélius par exemple, il est à deux reprises question des parties de l'âme (animi partes), soit la raison (ratio), le désir (libido) et la colère (iracundia $)^{35}$. De même Scipion, au livre I, déclare que «s'il peut y avoir dans l'âme humaine un pouvoir royal, ce sera l'autorité absolue d'un seul élément, sans aucun doute la raison ; c'est elle, en effet, qui est souveraine, il n'y a de place ni pour les passions, ni pour la colère, ni pour l'aveuglement (est enim animi pars optima; consilio autem dominante, nullum esse libidini, nullum irae, nullum temeritate locum) $\gg^{36}$. Ces passages ainsi que d'autres (on compte au total sept occurrences de l'expression pars animi, au singulier ou pluriel $)^{37}$ semblent en effet impliquer une tripartition raison-colère-désir (ratio-iracundia-libido) qui renvoie à la tripartition platonicienne ${ }^{38}$.

Au livre II, Scipion exprimait indirectement sa dette envers Platon comme suit : tandis que le dessein de celui-ci était de concevoir la cité « non pas telle qu'elle pût exister, mais telle que la théorie politique y fût mise en lumière. Moi, au contraire, si, du moins, je peux atteindre mon but, tout en suivant les mêmes principes qu'il a reconnus (rationibus eisdem quas ille vidit), je n'esquisserai pas une silhouette d'une cité imaginaire, mais en parlant d'un État très considérable [soit Rome] $\gg^{39}$. L'analogie entre individu-État apparaît ainsi comme l'un des principes (rationes) platoniciens auxquels Scipion fait ici allusion.

Mais il y a plus, car ces principes semblent comprendre, plus globalement, l'analogie entre l'ordre civique et l'ordre cosmique. En effet, l'analogie entre individu et État, associée à la doctrine des parties de l'âme, a chez Cicéron son pendant cosmologique : l'État est inséparable des principes de la Nature (naturae

35 ratio ibidini iracundiaque et ceteris vitiosis eiusdem partibus (III.xxiv); optima pars animi, id est sapientia, eius animi vitiosa imbecillasque partes, ut libidines, ut iracundias, ut perturbationes ceteras (III.xxv). Cf. Ferrary 1974, 765.

${ }^{36}$ I.xxxviii.

${ }^{37}$ Voici la liste de ces occurrences : I.38 (bis), II.40, III.24, 25 (ter).

${ }^{38}$ Cf. Tusc. I.20.

${ }^{39}$ II.xxx. S’il est vrai que le «réalisme» de Cicéron se distingue en cela de «l'idéalisme » de Platon, il convient néanmoins de rappeler que selon Scipion le régime monarchique est le meilleur régime en soi, tandis que le régime mixte est choisi dans la pratique uniquement pour des raisons de stabilité (I.xlv).

PLATO, The electronic Journal of the International Plato Society, n 11, 2011.

http://gramata.univ-paris1.fr/Plato/article100.html

(C) All rights of reproduction of any form reserved. 
RENAUD, François, «L'analogie platonicienne individu-État dans le De re publica de Cicéron : méthode antilogique et fondement de la justice ».

principia $)^{40}$. L'ordre naturel et hiérarchique est ancré en nous-mêmes, et peut être découvert par la réflexion. Comme il est dit dans le « Songe de Scipion » qui clôt le dialogue, la pensée (mens), principe divin en chacun de nous, gouverne le corps « comme (quam) ce dieu, qui occupe le premier rang, le fait pour le monde auquel nous appartenons ${ }^{41}$. D'où également la déclaration de Lélius qui en tire les conséquences morales : qui « refusera de lui obéir devra se fuir lui-même (ipse se fugiet $^{42}$ et, comme il a méprisé la nature humaine, il subira les plus cruels châtiments $\gg^{43}$.

La justice, selon Cicéron, est ancrée dans l'ordre de la nature. Il semble s'inspirer là encore de Platon et notamment du Timée, dont il sera d'ailleurs le traducteur. Comme il est dit expressément dans la République, les Idées existent par nature $(\phi \dot{v} \sigma \varepsilon l)^{44}$, et la cité véritable est conforme à la nature $(\kappa \alpha \tau \dot{\alpha} \phi \dot{v} \sigma \iota)^{45}$. Certes, Cicéron semble suivre, notamment dans le «Songe de Scipion », la conception stoïcienne de la nature comme monde sensible (mundus), tandis que selon l'ontologie platonicienne la nature n'est pas (d'abord) le monde sensible mais l'âme en tant que principe et réalité ultime. C'est pourquoi la tâche du politique chez Cicéron est d'imiter l'univers (mundum), tandis que celle-ci consiste chez Platon, plus exactement, à imiter les Idées ${ }^{46}$. Néanmoins, la doctrine stoïcienne du macrocosme-microcosme reste voisine de la doctrine platonicienne, selon laquelle la constitution de l'être humain correspond à celle de l'univers, l'un et l'autre étant composés d'un élément physique et d'une âme rationnelle ${ }^{47}$.

${ }^{40}$ III.iv ; cf. V.vi.

${ }^{41}$ VI.xxiv.

${ }^{42}$ Harold Tarrant me fait remarquer que ce passage rappelle la confession d'Alcibiade

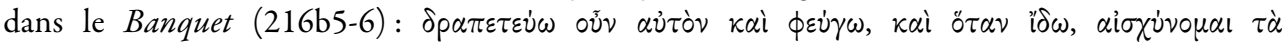
$\dot{\omega} \mu о \lambda o \gamma \eta \mu \dot{\varepsilon} v \alpha$. Dans ce cas le châtiment réside dans la conscience, douloureuse et durable, de l'infidélité à soi.

${ }^{43}$ III.xxii. $C f$. V.iii).

${ }^{44} \operatorname{Rép}$. 501b2-3.

${ }^{45}$ Rép. 428 e8-9. Cf. Pöschl 1936, 151-152.

${ }^{46}$ Cf. De leg. I.27 ; Tarrant 2006, 14-16.

${ }^{47}$ Cf. Philébe 28d-30d, en particulier Timée 29d-47e. Cf. Sharples 1986, 34-35.

PLATO, The electronic Journal of the International Plato Society, n 11, 2011.

http://gramata.univ-paris1.fr/Plato/article100.html

(C) All rights of reproduction of any form reserved. 


\section{Conclusion : le conflit théorie-pratique, la justice par-delà l'antilogie}

Cette seconde analogie entre l'ordre politique et l'ordre cosmique, qui englobe la première analogie, semble offrir à Cicéron une solution métaphysique au conflit entre théorie et pratique. La tension fondamentale entre théorie et pratique trouve son pendant platonicien dans le conflit entre sagesse et pouvoir, que la République dans son ensemble tente de surmonter. À la suite des préambules des livres I et III, le « Songe de Scipion » reprend cette question et insiste sur l'impérieuse nécessité d'une réconciliation entre ces deux exigences estimées également légitimes ${ }^{48}$. Ainsi l'étude théorique de l'ordre cosmique nous indique ce que doivent être l'ordre civique et la pratique morale.

La question du conflit entre théorie et pratique nous ramène, enfin, à l'antilogie des deux conceptions de la justice exposées dans la République: d'une part, la conception conventionnelle de la justice comme bien pour autrui, et d'autre part, la conception philosophique comme harmonie de l'âme. Il est significatif que Philus, dans son discours contre la justice, ne critique pas la conception spécifiquement platonicienne de la justice comme harmonie de l'âme : sa critique se limite à la conception de la justice comme bien pour autrui ${ }^{49}$. La raison en est assurément, en partie, que Cicéron comme auteur partage la conception platonicienne de la justice comme harmonie de l'âme et comme commandement de la raison. Dans sa défense de la monarchie, Scipion répond aux résistances de Lélius comme suit : «Pourquoi donc t'interroges-tu quant à ton sentiment sur l'État ? Si on confie à plusieurs les affaires publiques, il est évident qu'il n'y aura plus aucun pouvoir suprême; et si le pouvoir n'est pas un, il ne peut pas exister (nisi unum sit, esse nullum potest) $\gg^{50}$. Ainsi, la conception de la justice comme harmonie de l'âme et plus précisément comme le commandement de la raison constitue, dans le De re publica, un élément décisif du platonisme de Cicéron.

\footnotetext{
${ }^{48}$ Cf. Sharples 1986, 33 .

${ }^{49}$ Cf. Lévy 1992, 498.

${ }^{50}$ I.xxxviii.
}

PLATO, The electronic Journal of the International Plato Society, n 11, 2011.

http://gramata.univ-paris1.fr/Plato/article100.html

(C) All rights of reproduction of any form reserved. 
RENAUD, François, «L'analogie platonicienne individu-État dans le De re publica de Cicéron : méthode antilogique et fondement de la justice ».

\section{BIBLIOGRAPHIE}

BREGUET E. (1980), Cicéron, La République, tome I-II, [texte établi et traduit par E.B.], Paris.

FERRARY J.L. (1988), Philhellénisme et impéralisme, Rome.

(1974), « Le discours de Laelius dans le IIIe livre du De republica de Cicéron », Mélanges d'archéologie et d'histoire de l'École française de Rome 86, 1974, 745-771.

GLUCKER J. (1988), « Cicero's Philosophical Affiliations », J. Dillon et A. A. Long (dir.), The Question of «Eclecticism », Berkeley, 36-69.

HAHM D.E. (1999), « Plato, Carneades and Cicero's Philus (Cicero, rep. 3, 831) », Classical Quarterly 49, 167-183.

LÉVY C. (1992), « La Nouvelle Académie a-t-elle été antiplatonicienne ? », M. Dixsaut (dir.), Contre Platon, tome 1, Le platonisme dévoilé, Paris, 139156.

PÖSCHL V. (1936), Romischer Staat und griechisches Staatsdenken bei Cicero. Untersuchungen zu Ciceros Schrift De republica, Berlin (repr. 1962 Darmstadt).

POWELL J.G.F. (2006), M. Tulli Ciceronis De re publica, De legibus, Cato maior de senectute, Laelius de amicitia [texte établi par J.G.F.P.], Oxford.

SHARPLES R. (1986), « Cicero's Republic and Greek Political Theory », Polis 5 (1986), 30-50.

SLINGS S.R. (2003), Platonis Respublica [texte établi par S.R.S.], Oxford.

TARRANT H. (2006), « Platonic interpretation and eclectic theory », H. Tarrant et D. Baltzly (dir.), Reading Plato in Antiquity, Londres, 9-18.

ZETZEL J. E.G. (1995), Cicero. De re publica. Selections [texte édité et commenté par J.E.G.Z.], Cambridge.

PLATO, The electronic Journal of the International Plato Society, n 11, 2011.

http://gramata.univ-paris1.fr/Plato/article100.html

(C) All rights of reproduction of any form reserved. 